ARTÍCULO DE INVESTIGACIÓN

\title{
Jardines residenciales en Santiago de Chile: Extensión, distribución y cobertura vegetal
}

Residential gardens of Santiago, Chile: Extent, distribution and vegetation cover

\author{
SONIA REYES-PAECKE* \& LUIS MEZA
}

Instituto de Estudios Urbanos y Territoriales, Pontificia Universidad Católica de Chile,

El Comendador 1916, Santiago, Chile

*Autor correspondiente: sreyespa@uc.cl

\begin{abstract}
RESUMEN
Los jardines residenciales aportan una gran cobertura vegetacional en ambientes urbanos, pero han sido poco estudiados en América Latina. Este es el primer estudio sobre la materia realizado en el Área Metropolitana de Santiago (AMS). En esta ciudad los jardines residenciales cubren el $26 \%$ de la superficie urbana. Aunque la muestra analizada $(n=7236)$ evidencia una gran dispersión de tamaños, el $56 \%$ de los jardines mide entre 74 y $268 \mathrm{~m}^{2} . \mathrm{El} 37 \%$ de la superficie de los jardines está cubierta de árboles y arbustos, seguido por césped (34 \%), edificaciones menores $(26 \%)$ y solo un $3.0 \%$ carece de cobertura. Hay una alta correlación entre tamaño predial y cobertura vegetal $\left(\mathrm{R}^{2}\right.$ = 0.97), excepto en el centro de la ciudad, en donde a mayor tamaño predial se incrementa la superficie edificada y por tanto los jardines son pequeños. La cobertura vegetal total de los jardines residenciales alcanza 12000 hectáreas, equivalente al $19 \%$ del AMS, lo cual es 3.7 veces mayor a la superficie de áreas verdes públicas. Los factores más influyentes en la cobertura y estructura de la vegetación de los jardines, son el tamaño predial y la tipología de edificación.
\end{abstract}

Palabras clave: áreas verdes, cobertura del suelo, servicios ambientales, urbanización, vegetación urbana.

\begin{abstract}
Residential gardens provide important vegetation cover in urban environments but there is little research in Latin America. This is the first study on the subject in Santiago de Chile. In Santiago $26 \%$ of the urban area is covered by residential gardens. Even though there is a wide range of residential garden size, most of them measure between $74-$ $268 \mathrm{~m}^{2}$. The garden surface is mainly covered by trees and shrubs (37\%), followed by lawn (34\%), small buildings (26 $\%)$ while only $3 \%$ have no vegetation cover at all. A high correlation between lot size and vegetation cover was detected $\left(\mathrm{R}^{2}=0.97\right)$ except for the city centre where the increase in lot size is associated with an increase in the built up area. The vegetation cover of residential gardens reaches 12000 hectares (19\% of the urban area) which surpasses that of public green spaces. Both lot size and building typology are the most important factors influencing cover and structure of vegetation in residential gardens.
\end{abstract}

Key words: environmental services, green spaces, land cover, urbanization, urban vegetation.

\section{INTRODUCCIÓN}

Los estudios que han evaluado la cobertura, distribución y abundancia de la vegetación urbana en el Área Metropolitana de Santiago han considerado solamente la vegetación presente en espacios públicos (De la Maza et al. 2002, Escobedo et al. 2006, Hernández 2007), al igual que los estudios referidos a la presencia de aves nativas en la ciudad (Urquiza \& Mella 2002, Díaz \& Armesto 2003, Mella \& Loutit 2007). Hasta ahora no hay publicaciones referidas a los jardines residenciales y no se cuenta con una estimación de la superficie ocupada por ellos. Probablemente la ausencia de estudios se debe a la condición de propiedad privada y el alto grado de fragmentación, condiciones que dificultan el estudio de sus características y composición (Gaston et al. 2005).

Estudios realizados en diversas ciudades (e.g., Sheffield, Belfast, Cardiff, Edinburgh, Leicester y Oxford, UK; Lauris, Francia; São Luis, Brasil; San Rafael Coxcatlán, México) muestran que los jardines residenciales 
contribuyen a incrementar la cobertura vegetal, densidad arbórea y biodiversidad urbana, y pueden contribuir a la conservación de la biodiversidad nativa, ya que contienen especies que están ausentes en zonas rurales (Blanckaert et al. 2004, Gaston et al. 2005, Smith et al. 2005, Loram et al. 2008, Marco et al. 2010, Akinnifesi et al. 2010).

Asimismo, se ha demostrado que las características de los jardines urbanos y de la vegetación presente en ellos están influenciadas por un conjunto de factores sociales y culturales, siendo los más importantes el nivel de ingresos de los residentes, el tamaño de los jardines, la densidad de edificación, la tipología de viviendas y las preferencias de los propietarios (Blanckaert et al. 2004, Albuquerque et al. 2005, Kinzig et al. 2005, Smith et al. 2005, Daniels \& Kirkpatrick 2006, Loram et al. 2008, Marco et al. 2010). Las condiciones climáticas y del suelo influyen en la diversidad de especies y complejidad estructural de los jardines y en las técnicas de manejo aplicadas por los propietarios (Wezel \& Bender 2003, Marco et al. 2010).

La composición de la biodiversidad urbana (riqueza de especies) depende de factores sociales, culturales y económicos, los cuales, en muchos casos, superan la influencia de los factores ecológicos (ver De la Maza et al. 2002, Kinzig et al. 2005, Barbosa et al. 2007, Marco et al. 2010). Sin embargo, la complejidad de la dinámica espacial y temporal de las ciudades hace difícil establecer relaciones causales simples entre la biodiversidad urbana y los factores antes mencionados. Por esta razón, algunos autores han propuesto un enfoque conceptual basado en el reconocimiento de procesos bottom-up y top-down (Kinzig et al. 2005, Marco et al. 2010): los primeros involucran decisiones o acciones de pequeña escala, realizadas por individuos o familias, tales como la elección de especies para el jardín de la vivienda; en tanto los procesos top-down se refieren a las decisiones y estrategias de la administración o de las autoridades urbanas, tales como la planificación de los usos del suelo o la selección de especies para los parques y espacios públicos.

El presente estudio adopta este enfoque, analizando un proceso bottom-up como es la decisión respecto a la cobertura vegetal de los jardines de las viviendas, y busca establecer correlaciones entre estas decisiones y el nivel de ingresos de la población. Ya se ha establecido la correlación entre las características socioeconómicas de la población y la vegetación de espacios públicos del AMS (De la Maza et al. 2002, Figueroa 2009, Reyes-Paecke \& Figueroa 2010), así como la relación entre nivel de ingresos del municipio y la vegetación de espacios públicos del AMS (Escobedo et al. 2006), pero en ambos casos se refiere al nivel de decisión de la administración municipal y por tanto corresponden a procesos top-down.

El objetivo del estudio ha sido caracterizar la superficie y cobertura vegetal de los jardines residenciales del Área Metropolitana de Santiago (AMS) y establecer patrones de distribución de la vegetación presente en ellos. Dado que no existen estudios previos, esta primera investigación se ha concentrado en: (i) medición de la superficie y distribución de los jardines dentro del AMS, (ii) medición de la cobertura vegetal presente en ellos, y (iii) establecer correlaciones con variables conocidas como el nivel de ingresos de la población y tipologías de edificación. En una segunda etapa se analizará la composición de especies y las preferencias de los propietarios.

\section{MÉTODOS}

\section{Área de estudio}

El Área Metropolitana de Santiago (AMS) está localizada en $\operatorname{los} 33^{\circ} \mathrm{S}$ en la Región Mediterránea de Chile, la cual ha sido calificada como un sitio prioritario para la conservación de la biodiversidad global debido a la alta tasa de endemismo de la flora y fauna (Arroyo et al. 1999). La ciudad tiene aproximadamente 5.4 millones de habitantes (INE 2004) y la superficie urbana supera levemente las 64000 hectáreas $\left(640 \mathrm{~km}^{2}\right)$, con una densidad poblacional promedio de 84 habitantes/ hectárea. Hay grandes diferencias socioeconómicas entre las distintas comunas que forman la ciudad, debido al alto grado de segregación espacial existente entre grupos con diferentes niveles de ingreso (Rodríguez 2008).

\section{Información básica y fotointerpretación}

El análisis se realizó sobre un mosaico de ortofotos del Servicio Aerofotogramétrico de la Fuerza Aérea (SAF), de 2006. Sobre dicho mosaico se superpuso un plano base de manzanas del AMS con usos de suelo predominantes y tamaño predial promedio por manzana, cuyas fuentes de información fueron: población por manzana según Censo 2002, del Instituto Nacional de Estadísticas, manzanas urbanas del proyecto "Sistema de Indicadores Comunales" (SIDICO) de la Secretaría Regional Ministerial de Vivienda y Urbanismo (SEREMI 
MINVU), permisos de edificación años 2002 y 2005 y datos de la Encuesta Nacional de Estratificación Social (CASEN) del año 2006. Se distinguieron usos de suelo residencial, industrial, comercial, agrícola y otros usos. Para obtener la muestra de jardines, se trazó una grilla sobre el mosaico del AMS y se definieron puntos de muestreo de $500 \mathrm{~m}$ de radio, al interior del cual se digitalizaron todos los predios y jardines residenciales (Fig. 1). Para el estudio se entiende por predio todo sitio, lote o terreno de propiedad pública o privada, excluidos los espacios públicos (MINVU 2011). Los jardines residenciales se definen como el espacio localizado alrededor de la vivienda, en el cual los propietarios distribuyen espacial y temporalmente vegetación y

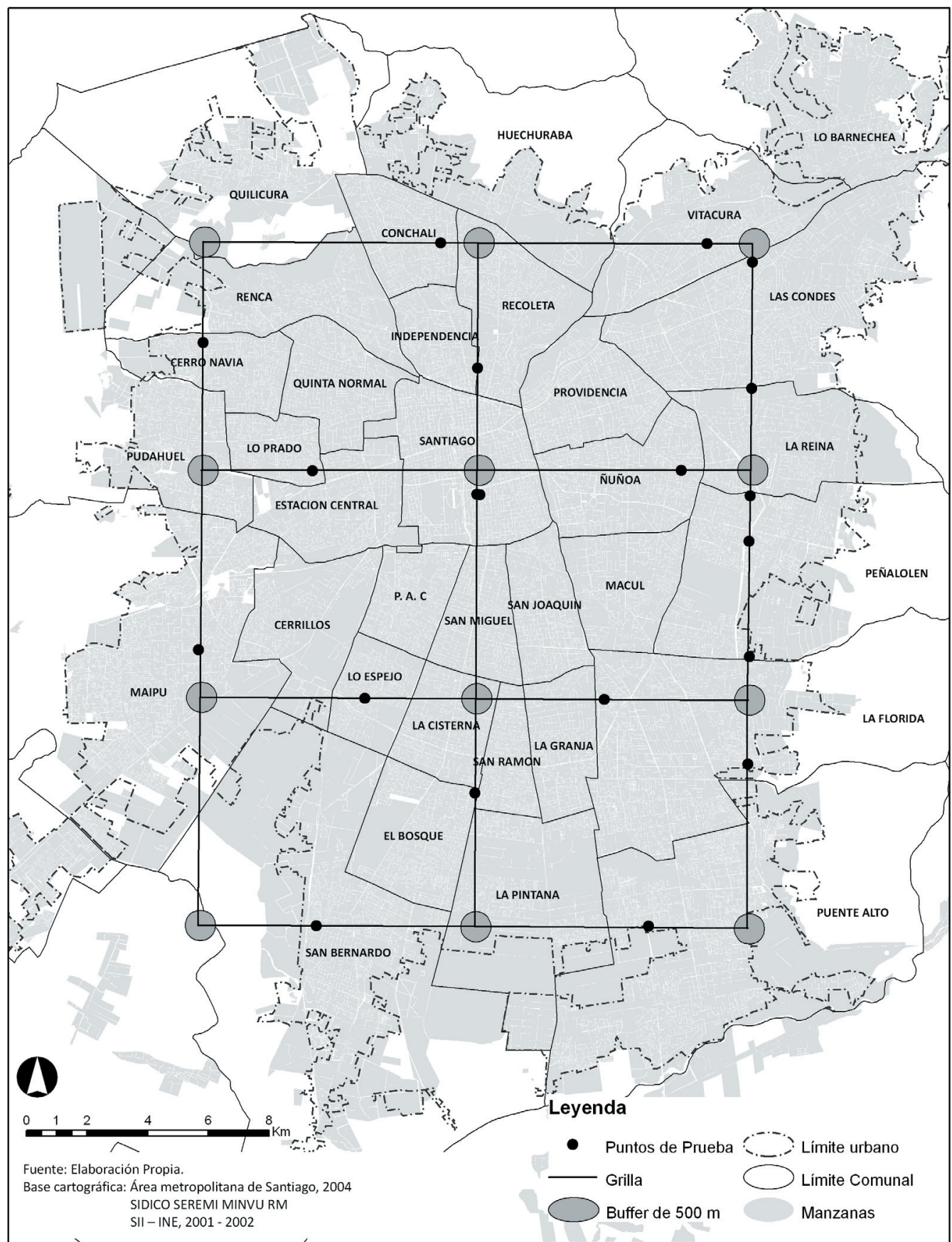

Fig. 1: Ubicación de los puntos de muestreo en el Área Metropolitana de Santiago. Fuente: elaboración propia basado en Meza (2009).

Location of sampling points in the Santiago Metropolitan Area. 
otros elementos destinados al descanso, recreación o actividades tales como cultivos agrícolas $\mathrm{u}$ ornamentales de pequeña escala. Para su medición se define como la superficie del predio residencial menos la superficie ocupada por la vivienda, en metros cuadrados (Meza 2009, Marco et al. 2010). La manzana urbana consiste en un agregado de predios de propiedad pública o privada, delimitado por calles, que constituye la unidad mínima de información censal.

Se realizó un control de terreno en los diez puntos analizados, identificando los predios digitalizados, el uso del suelo y la longitud del frente predial. Con ello se validó la información contenida en las bases de datos utilizadas. Toda la información fue georreferenciada utilizando $\mathrm{ArcView}^{\circledR} 3.2$ y ArcGIS ${ }^{\circledR}$ 9.2. Los resultados fueron extrapolados al AMS, basado en la alta correlación entre el tamaño de jardín y tamaño predial $\left(R^{2}=0.78\right)$, exceptuando la comuna de Santiago, en donde la correlación es baja $\left(\mathrm{R}^{2}=0.50\right)$. Debido a esta diferencia se realizaron extrapolaciones diferenciadas para la comuna de Santiago y resto del AMS, mediante ArcGIS ${ }^{\circledR}$ 9.2, sobre el plano base de manzanas del AMS que contiene el tamaño predial predominante en la manzana. La validez de la regresión se verificó en 300 jardines localizados fuera de los puntos de muestreo, sobre los ejes de la grilla (ver Fig. 1). Los resultados se integraron en un solo mapa que muestra la distribución de los jardines residenciales del AMS, por rango de tamaño, mediante $\operatorname{ArcGIS}^{\circledR}$ 9.2.

Se analizaron separadamente los jardines unifamiliares de los colectivos, para identificar las diferencias entre ambas tipologías de vivienda. Los primeros corresponden a predios que contienen viviendas aisladas, pareadas o continuas, en donde el jardín tiene un único propietario. Los jardines colectivos corresponden a predios con edificios de departamentos, en donde el jardín es compartido por todos los propietarios. Las características de cada punto de muestreo se resumen en la Tabla 1.
La cobertura del suelo de los jardines se determinó mediante fotointerpretación. La cobertura se clasificó en cuatro tipos: césped (herbáceas y césped), árbol o arbusto (vegetación arbustiva y arbórea), construido (terraza, piscina, cobertizo y otras instalaciones similares) y sin cobertura (sin vegetación ni construcciones). Se obtuvo el porcentaje de cada tipo de cobertura para cada rango de tamaño del jardín. Los resultados fueron extrapolados al AMS sobre el plano base de manzanas, con ArcGIS ${ }^{\circledR}$ 9.2. El número y superficie de jardines por punto de muestreo se presentan en la Tabla 2.

La composición socioeconómica de la población se obtuvo a partir de la clasificación del nivel de ingresos de los hogares del AMS, expresada en el grupo socioeconómico (GSE) predominante por manzana. La población se divide en cinco grupos: $\mathrm{ABC} 1, \mathrm{C} 2, \mathrm{C} 3, \mathrm{D}$ y E, según metodología ADIMARK (http://www.adimark. cl/medios/estudios/Mapa_Socioeconomico_de_Chile. pdf), siendo $\mathrm{ABC} 1$ el grupo de más altos ingresos y grupo $\mathrm{E}$, el de más bajos ingresos. Mediante $\operatorname{ArcGIS}^{\circledR} 9.2$ se superpusieron los mapas del GSE y jardines residenciales por manzana, en la misma proyección y escala.

\section{RESULTADOS}

\section{Tamaño de los jardines}

Los 7236 jardines analizados suman una superficie total de 111.4 hectáreas. El tamaño promedio es de $154 \mathrm{~m}^{2}$, con una desviación estándar de $281.4 \mathrm{~m}^{2}$. El jardín de menor tamaño mide solo $8 \mathrm{~m}^{2}$ y el de mayor tamaño es de $9499 \mathrm{~m}^{2}$. La distribución es asimétrica

TABLA 1

Usos de suelo, superficie residencial y superficie total de jardines en los puntos de muestreo.

Land uses, residential surface, and total garden surface in sampling points.

\begin{tabular}{lcccc}
\hline Punto de muestreo & Usos de suelo & $\begin{array}{c}\text { Superficie } \\
\text { residencial }\left(\mathrm{m}^{2}\right)\end{array}$ & $\begin{array}{c}\text { Superficie total } \\
\text { jardín }\left(\mathrm{m}^{2}\right)\end{array}$ & $\begin{array}{c}\text { Superficie jardín/ } \\
\text { superficie residencial (\%) }\end{array}$ \\
\hline Maipú & $\mathrm{R}, \mathrm{I}$ & 194000 & 36568 & 18.8 \\
Pudahuel & $\mathrm{R}, \mathrm{I}$ & 465000 & 134925 & 29.0 \\
La Pintana & $\mathrm{R}, \mathrm{A}$ & 403000 & 60481 & 15.0 \\
La Cisterna & $\mathrm{R}, \mathrm{C}$ & 677000 & 181847 & 26.9 \\
Santiago Centro & $\mathrm{C}, \mathrm{I}, \mathrm{R}$ & 545000 & 23575 & 4.3 \\
Recoleta & $\mathrm{R}, \mathrm{I}$ & 657000 & 45232 & 6.9 \\
Puente Alto & $\mathrm{R}$ & 104000 & 56830 & 54.6 \\
La Florida & $\mathrm{R}$ & 400000 & 83685 & 20.9 \\
La Reina & $\mathrm{R}$ & 595000 & 307619 & 51.7 \\
Vitacura- Las Condes & $\mathrm{R}$ & 529000 & 183533 & 34.7 \\
\hline
\end{tabular}

Usos de suelo: $\mathrm{R}$ = residencial, $\mathrm{I}$ = industrial, $\mathrm{C}$ = comercial, $\mathrm{A}=$ agrícola.

Fuente: elaboración propia basado en Meza (2009). 
con concentración de datos hacia la izquierda del promedio. El índice de Gini muestra una baja concentración de los datos, con un valor de 0.254 .

La muestra incluye espacios sociales y geográficos muy diversos, lo cual se refleja en la variedad de tamaños de los jardines y en las diferencias en el número y superficie total de jardines por punto de muestreo (Tabla 2). Los resultados son un reflejo de los tipos de urbanización predominantes en el AMS: los jardines medianos son los más importantes tanto en número como en superficie. Los jardines grandes y muy grandes son los más escasos, pero representan más del $40 \%$ de la superficie total de jardines del AMS. En cambio, los jardines pequeños son más numerosos pero su aporte en superficie apenas supera el $10 \%$ (Tabla 3).

Los jardines de tamaño mediano muestran una amplia distribución en el AMS, aunque se concentran especialmente en el sector poniente de la ciudad. En cambio, los jardines de tamaño pequeño se concentran en aquellos sectores del AMS en donde predominan las viviendas sociales (viviendas construidas con financiamiento estatal y destinadas a familias de bajos ingresos): parte de las comunas de Maipú, Pudahuel, Renca, El Bosque, La Pintana, La Florida y Puente Alto. También se encuentran, aunque en menor cantidad, en las comunas de La Granja, Pedro Aguirre Cerda, San Miguel, Recoleta, Independencia, Conchalí y Lo Barnechea. Por último hay una fuerte

TABLA 2

Número y superficie de jardines residenciales por punto de muestreo, AMS.

Number, garden surface and garden size in sampling points.

\begin{tabular}{lccccc}
\hline Punto de muestreo & $\begin{array}{c}\text { Número de } \\
\text { jardines }\end{array}$ & $\begin{array}{c}\text { Jardín más } \\
\text { grande }\left(\mathrm{m}^{2}\right)\end{array}$ & $\begin{array}{c}\text { Jardín más } \\
\text { pequeño }\left(\mathrm{m}^{2}\right)\end{array}$ & $\begin{array}{c}\text { Tamaño promedio } \\
\text { jardín }\left(\mathrm{m}^{2}\right)\end{array}$ & $\begin{array}{c}\text { Desviación } \\
\text { estándar }\end{array}$ \\
\hline Maipú & 236 & 1222 & 10 & 155 & 133.5 \\
Pudahuel & 1391 & 4408 & 10 & 97 & 172.2 \\
La Pintana & 80 & 9499 & 13 & 756 & 1702.2 \\
La Cisterna & 753 & 5315 & 11 & 242 & 362.6 \\
Santiago Centro & 185 & 1794 & 12 & 128 & 257.0 \\
Recoleta & 546 & 1251 & 8 & 83 & 119.6 \\
Puente Alto & 839 & 314 & 10 & 68 & 32.4 \\
La Florida & 890 & 1431 & 12 & 94 & 85.4 \\
La Reina & 1377 & 6079 & 34 & 223 & 259.5 \\
Vitacura-Las Condes & 939 & 1453 & 47 & 196 & 133.0 \\
\hline
\end{tabular}

Fuente: elaboración propia basada en Meza (2009).

TABLA 3

Tamaño de los jardines en la muestra analizada.

Garden size of analyzed sample.

\begin{tabular}{lccccc}
\hline Rango de tamaño & Tamaño $\left(\mathrm{m}^{2}\right)$ & Número & $\begin{array}{c}\text { Porcentaje del } \\
\text { total de jardines }\end{array}$ & $\begin{array}{c}\text { Superficie } \\
\left(\mathrm{m}^{2}\right)\end{array}$ & $\begin{array}{c}\text { Porcentaje de la superficie } \\
\text { total de jardines }(\%)\end{array}$ \\
\hline Pequeño & $<74$ & 2423 & 33.5 & 122045 & 11.0 \\
Mediano & $74-268$ & 4052 & 56.0 & 541765 & 48.6 \\
Grande & $269-966$ & 692 & 9.6 & 299543 & 26.9 \\
Muy Grande & $>966$ & 69 & 1.0 & 150942 & 13.5 \\
Total muestra & & 7236 & 100.0 & 1114295 & 100.0 \\
\hline
\end{tabular}

Fuente: elaboración propia basado en Meza (2009). 
presencia de jardines pequeños en el centro de la ciudad producto del alto porcentaje de ocupación del suelo (Fig. 2).

Los jardines grandes se concentran en las comunas de la zona oriente: Ñuñoa, La Reina, Providencia, Las Condes, Vitacura y
Lo Barnechea. Aunque también se observan importantes concentraciones en La Florida, Macul, San Miguel, Cerrillos y en el centro de Maipú. Los jardines de mayor tamaño (> 966 $\mathrm{m}^{2}$ ) se encuentran en la periferia oriente del AMS, en las comunas La Florida, Peñalolén, La

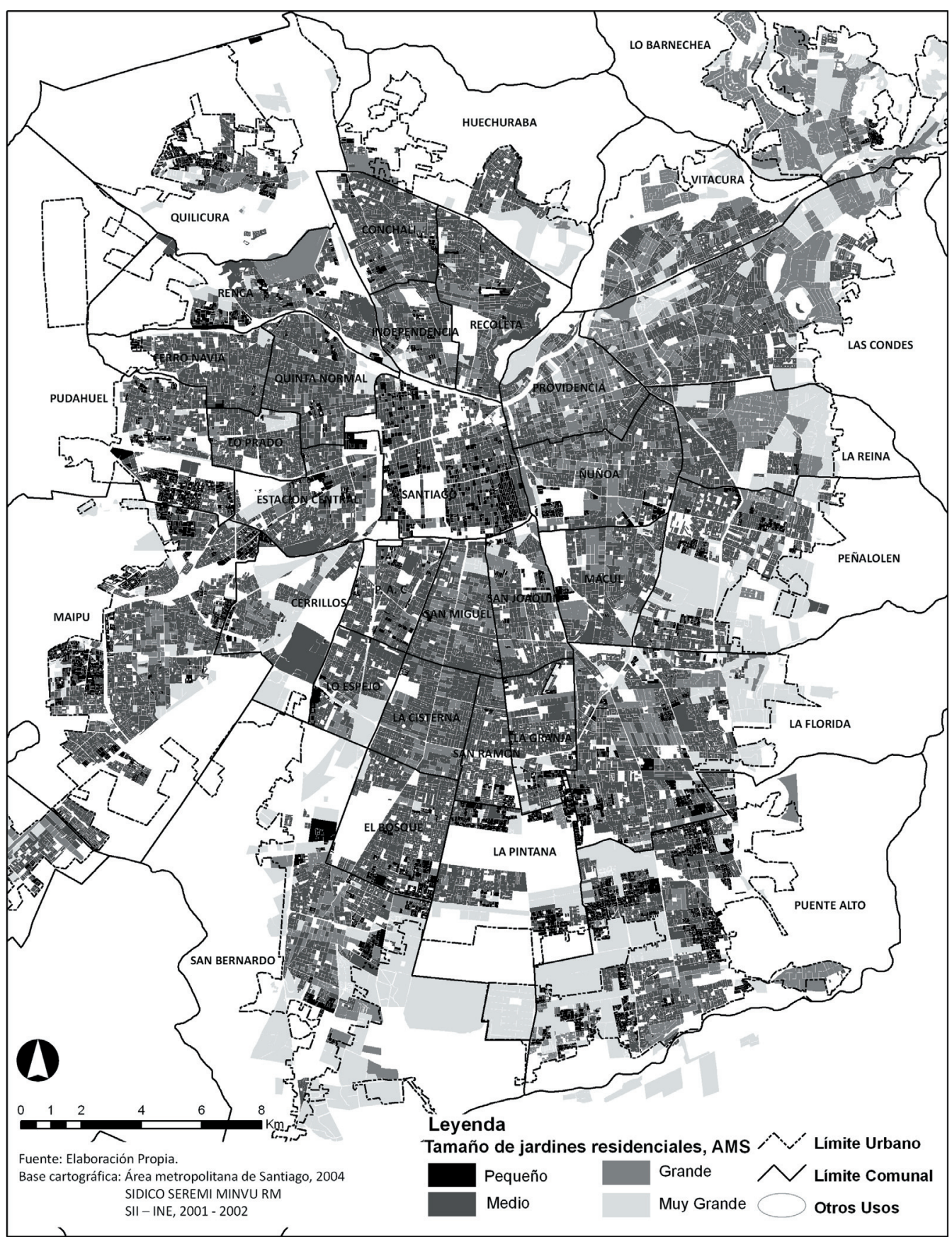

Fig. 2: Tamaño de los jardines residenciales en el Área Metropolitana de Santiago. Fuente: Elaboración propia. 
Reina, Las Condes, Vitacura y Lo Barnechea. También se observan agrupaciones de jardines de este tamaño en las comunas de Puente Alto, La Pintana y San Bernardo, correspondientes a un tipo de parcelación de los años 1950 y 1960 llamados huertos familiares (Fig. 2).

En la muestra se registraron 205 jardines colectivos que suman 12.1 hectáreas, mientras que los jardines unifamiliares son 7031 con una superficie total de 99.3 hectáreas. Ello responde a la predominancia de viviendas unifamiliares en la estructura urbana del AMS. Los jardines unifamiliares son mayoritariamente de tamaño mediano y pequeño, asociados a los tamaños prediales más frecuentes en el AMS, como se muestra en la Tabla 4. En el caso de los jardines colectivos, son más numerosos los jardines grandes, seguido por los jardines medianos y muy grandes. Los jardines grandes y muy grandes aportan poco más del $90 \%$ de la superficie de jardines colectivos (Tabla 4).

\section{Correlación entre tamaño predial y tamaño de jardin}

La muestra de jardines permitió evidenciar la alta correlación entre el tamaño predial y el tamaño del jardín en el AMS $\left(\mathrm{R}^{2}=0.715\right)$, exceptuando solo a la comuna de Santiago (centro histórico de la ciudad) en donde no se cumple dicha correlación. Por el contrario, el mayor tamaño predial está asociado a una mayor superficie edificada, lo cual es propio de los centros de las grandes ciudades, que se caracterizan por una mayor densidad de edificación (Forman 2008).

La más alta correlación entre tamaño predial-tamaño jardín se encontró en la zona oriente $\left(\mathrm{R}^{2}=0.97\right)$ y sur del AMS $\left(\mathrm{R}^{2}=0.88\right)$, seguidos por la zona norte $\left(\mathrm{R}^{2}=0.60\right)$ y poniente $\left(R^{2}=0.62\right)$ en donde alcanza valores más bajos, pero sigue siendo significativa. Estos resultados confirman la existencia de un gradiente de densidad de edificación y superficie de jardín, desde el centro de la ciudad hacia la periferia, con el centro más denso e impermeable, hacia una periferia menos densa y con mayor superficie destinada a jardines. Este gradiente se registra con particular claridad hacia la zona oriente del AMS, en cuya periferia predominan los predios de gran tamaño con jardines igualmente grandes. En el resto de la periferia, la presencia de poblaciones de vivienda social, incrementa la densidad de edificación en los bordes de la ciudad (ver Fig. 2), dejando sectores con menor densidad de edificación y mayor superficie de jardines en la franja intermedia.

\section{Cobertura del suelo en los jardines residenciales}

Los jardines residenciales de Santiago se caracterizan por una importante cobertura de vegetación en todos los rangos de tamaño (Fig. 3). Para el total de jardines la suma de cobertura de césped y árbol o arbusto supera el $70 \%$ de la superficie, inclusive en los jardines pequeños. Las diferencias existentes entre los

TABLA 4

Número y superficie de jardines en viviendas unifamiliares y edificios de departamentos, según rango de tamaño.

Number and surface of gardens of single-family housing and apartment buildings, by size rank.

\begin{tabular}{lcccccccc}
\hline \multirow{2}{*}{$\begin{array}{l}\text { Rangos de } \\
\text { tamaño }\end{array}$} & \multicolumn{3}{c}{ Jardines en viviendas unifamiliares } & \multicolumn{2}{c}{ Jardines en edificios de departamentos } \\
\cline { 2 - 9 } & \multicolumn{2}{c}{ Número } & \multicolumn{2}{c}{ Superficie } & \multicolumn{2}{c}{ Número } & \multicolumn{2}{c}{ Superficie } \\
\cline { 2 - 9 } & $\mathrm{N}$ & $\%$ & $\mathrm{~m}^{2}$ & $\%$ & $\mathrm{~N}$ & $\%$ & $\mathrm{~m}^{2}$ & $\%$ \\
\hline Pequeño & 2412 & 34.0 & 121642 & 12.1 & 11 & 5.0 & 403 & 0.3 \\
Medio & 3990 & 57.0 & 530769 & 53.5 & 62 & 30.0 & 10996 & 9.1 \\
Grande & 590 & 8.4 & 249776 & 25.2 & 102 & 50.0 & 49767 & 41.1 \\
Muy Grande & 39 & 0.6 & 91119 & 9.2 & 30 & 15.0 & 59822 & 49.4 \\
Total & 7031 & 100.0 & 993306 & 100.0 & 205 & 100.0 & 120987 & 100.0 \\
\hline
\end{tabular}

Fuente: elaboración propia basado en Meza (2009). 
distintos tamaños de jardín se expresan en la proporción de cobertura vegetal respecto de las construcciones menores y del suelo desnudo. Mientras los jardines pequeños tienen el $57 \%$ de superficie ocupado por vegetación y un 42.2
\% con algún tipo de construcción, los jardines medianos presentan un $68.7 \%$ de superficie con vegetación, un $30.1 \%$ construido y solo un $1.2 \%$ carece de cobertura.

La superficie total estimada de jardines

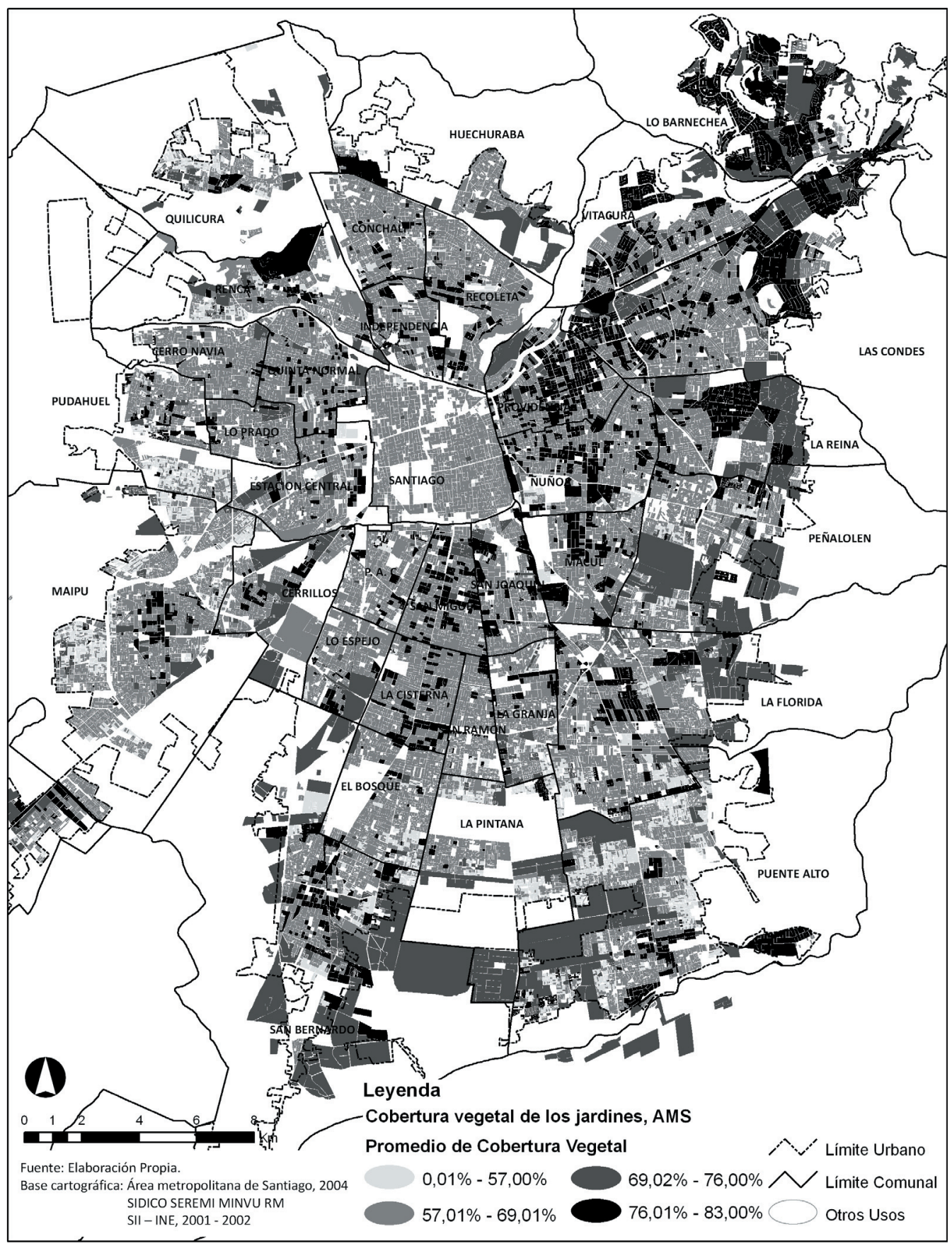

Fig. 3: Cobertura vegetal de los jardines residenciales en Área Metropolitana de Santiago. Fuente: Elaboración propia basado en Meza (2009).

Vegetation cover of residential gardens in Santiago Metropolitan Area. 
TABLA 5

Superficie y cobertura vegetal estimada en jardines residenciales del AMS.

Garden size and vegetation cover in residential gardens of AMS.

\begin{tabular}{lccccc}
\hline Rango de tamaño & Pequeño & Mediano & Grande & Muy grande & Total \\
\hline Superficie total de jardines residenciales (hectáreas) & 1285.3 & 8234.8 & 3293.2 & 3896.4 & 16709.6 \\
Porcentaje de la superficie total de jardines (\%) & 7.7 & 49.3 & 19.7 & 23.3 & 100.0 \\
\% con respecto a la superficie total del AMS & 20 & 12.8 & 5.1 & 6.1 & 26.0 \\
Superficie con cobertura vegetal (hectáreas) & 732.6 & 5682.0 & 2733.3 & 2961.2 & 12109.2 \\
\% respecto cobertura vegetal total de jardines del AMS (\%) & 6.1 & 46.9 & 22.6 & 24.5 & 100.0 \\
\% cobertura vegetal respecto superficie total del AMS & 1.10 & 8.80 & 4.20 & 4.60 & 18.80 \\
\hline
\end{tabular}

Fuente: elaboración propia basado en Meza (2009).

residenciales en el AMS alcanza las 16710 hectáreas, equivalentes al $26 \%$ de la superficie urbana. El $72.5 \%$ de dicha superficie está cubierta de vegetación (árbol-arbusto y césped), equivalentes a 12.109 hectáreas. Por tanto, los jardines residenciales representan una importante reserva de suelo permeable y de vegetación en el AMS, especialmente los jardines medianos. Estos son también los más relevantes términos de servicios ambientales por su amplia distribución en la ciudad, a diferencia de los jardines grandes y muy grandes, que aportan una superficie vegetal similar pero están concentrados en la zona oriente y sur del AMS (Tabla 5).

Los jardines colectivos muestran una predominancia de cobertura arbórea-arbustiva (48.3\%) por sobre el césped (26\%), a diferencia de los jardines unifamiliares en donde ambas coberturas son muy parecidas (31 y $35.8 \%$ ).

Nivel de ingresos de la población, tamaño y cobertura vegetacional de jardines

La población del AMS pertenece mayoritariamente a GSE medios y medio-bajos. El 38 \% de los jardines analizados pertenece a familias del GSE C3, quienes aportan el $29 \%$ de la superficie total de jardines, mientras que el $33 \%$ de los jardines pertenece a familias del GSE C2, quienes aportan el $37 \%$ de la superficie total de jardines de Santiago (Meza 2009). El $17 \%$ de los jardines pertenece al GSE D, los cuales contribuyen con el $19 \%$ de la superficie total de jardines (Meza 2009). El grupo de más bajos ingresos está asociado a predios de pequeño tamaño y a edificios de viviendas sociales, los cuales en su gran mayoría carecen de jardín, razón por la cual contribuyen con una porción menor de la superficie de jardines del AMS. Hay una gran coincidencia espacial entre el GSE $\mathrm{ABC} 1$ (los más altos ingresos) y los jardines de mayor tamaño (grande y muy grande), ya que ambos se concentran en el sector oriente de la ciudad. En esta misma zona se concentran las áreas verdes -públicas y privadas- de mayor tamaño y cobertura vegetal (Figueroa 2009, Reyes-Paecke \& Figueroa 2010) y la cobertura arbórea urbana (De la Maza et al. 2002), lo cual refuerza la desigual distribución de la vegetación en el AMS.

\section{DISCUSIÓN}

\section{Estructura urbana y jardines en el AMS}

Siendo este el primer trabajo que evalúa la superficie y cobertura vegetal de los jardines residenciales en el AMS, permite establecer algunos aspectos poco conocidos de la estructura urbana, que tienen importancia ecológica: los jardines residenciales constituyen la principal reserva de vegetación urbana $\mathrm{y}$ suelo permeable del AMS, al igual que en las ciudades inglesas analizadas por Loram et al. (2008), pero el tamaño promedio de los jardines del AMS es significativamente menor, por tanto se trata de un recurso bastante más fragmentado. Un hallazgo importante es la predominancia de vegetación en todos los tamaños de jardín, inclusive en aquellos de 
menor tamaño $\left(<74 \mathrm{~m}^{2}\right)$, los cuales aportan los servicios ambientales de la vegetación en las zonas más densamente pobladas de la ciudad, que coincidentemente cuentan con escasas áreas verdes públicas (Meza 2009, ReyesPaecke \& Figueroa 2010).

Un patrón documentado en otras ciudades es la correlación entre el nivel de ingresos de la población y el tamaño del jardín, lo cual es corroborado en Santiago. También se ha encontrado una alta correlación entre el nivel de ingresos y la diversidad estructural de los jardines (Kinzig et al. 2005, Loram et al. 2008), no obstante, en el presente estudio la evidencia no es concluyente. Por una parte, dicha relación está mediada por el tamaño del jardín, ya que a mayor superficie es posible incluir una mayor diversidad de estratos. Por otra parte, hay una limitación asociada a la metodología utilizada (fotointerpretación): en un jardín pequeño la cobertura arbórea puede ocultar los estratos arbustivos y herbáceos, de manera que el presente estudio no permite concluir respecto de esta correlación.

En cuanto a la influencia de la tipología de vivienda, se registra una mayor cobertura arbórea-arbustiva en los jardines colectivos (48.3\%).

Una importante diferencia entre Santiago y otras ciudades reportadas en la literatura, es la existencia de diferentes gradientes de densidad vegetacional en la misma aglomeración urbana: en el cuadrante nororiente del AMS, en donde se localiza la población de más altos ingresos, se observa un incremento gradual del tamaño predial y la cobertura vegetal hacia el límite urbano, en donde se concentran los jardines grandes y muy grandes (Fig. 3). En el resto de la ciudad el gradual incremento de cobertura vegetal desde el centro hacia la periferia, es interrumpido por la presencia de zonas densamente edificadas y localizadas en las cercanías del límite urbano (Fig. 3). Esta alta densidad de la urbanización periférica extrema la diferencia entre el espacio urbano y rural, estableciendo un brusco cambio en la cobertura del suelo.

\section{Procesos top-down y bottom-up}

La decisión de mantener vegetación en los jardines de las viviendas así como la selección de especies, corresponde a un proceso bottomup, que ocurre en la esfera de las decisiones privadas, pero tiene un importante impacto en la esfera pública, ya que la sumatoria de las coberturas vegetales de los jardines urbanos supera 3.7 veces la superficie de áreas verdes públicas del AMS. En esta ciudad, las familias prefieren contar con vegetación aun cuando el jardín sea pequeño, lo cual es un indicador muy positivo desde el punto de vista de los beneficios para la ciudad (estéticos, climáticos, ecológicos). Sin embargo, las familias están limitadas por el tamaño predial, dentro del cual pueden mantener su jardín.

La estructura predial (tamaño, disposición espacial) está determinada por condicionantes mercantiles (precio del suelo urbano), normativas (regulación de tamaño mínimo predial) y de políticas de vivienda (tamaño y localización de viviendas sociales). Todos ellos corresponden a procesos top-down. Por tanto la posibilidad de incrementar la masa vegetacional total de Santiago al interior de los jardines residenciales, depende también de decisiones de las esferas de administración urbana comunal (Municipalidad), regional (Gobierno Regional y Secretarías Regionales Ministeriales) y nacional (Ministerio de Vivienda y Urbanismo).

En las comunas de más bajos ingresos, los tamaños prediales son pequeños e impiden la existencia de grandes jardines en las viviendas y por tanto la vegetación está más fragmentada y la presencia de árboles es menor (De la Maza et al. 2002, Hernández 2007). Adicionalmente, los municipios de estas comunas disponen de escasos recursos para la mantención de áreas verdes y vegetación (Escobedo et al. 2006, Reyes-Paecke \& Figueroa 2010), lo cual resulta en espacios públicos con menor cobertura vegetal, árboles de menor tamaño y deficiente nivel de mantención.

En consecuencia, la convergencia de procesos top-down y bottom-up amplifica las diferencias de cobertura vegetacional entre las comunas del AMS y confirma la predominancia de factores socioeconómicos por sobre los ecológicos en la distribución y estructura de la vegetación urbana.

\section{Implicancias para las políticas urbanas}

Algunas políticas urbanas han influido de manera determinante en los patrones observados. La comuna de Santiago ha 
sido objeto de una sostenida política de densificación residencial, destinada a evitar la pérdida de población (Ilustre Municipalidad de Santiago 2006). Uno de sus resultados ha sido el reemplazo de antiguas viviendas con jardín interior, por edificios multifamiliares carentes de jardín. Con ello, se ha incrementado la densidad poblacional pero se han perdido los jardines interiores y la vegetación que contenían. Para evitar este negativo impacto, las iniciativas de densificación de los barrios centrales deben cautelar la permanencia de jardines con vegetación, ya que constituye uno de los elementos clave para el bienestar de la población y también es un componente relevante de las estrategias de adaptación a las consecuencias del cambio climático.

La existencia de jardines de mayor tamaño en la periferia este, colindando con los espacios silvestres protegidos de la Cordillera de Los Andes, establece una alta continuidad de la cobertura arbórea entre el área urbana y rural, lo cual puede favorecer la presencia de aves que habitan en los árboles, ya que pueden percibir el follaje como un hábitat continuo por sobre las edificaciones urbanas (Díaz \& Armesto 2003). Se ha documentado la presencia de aves nativas en zonas residenciales de Santiago, asociadas a la cobertura arbórea (Díaz \& Armesto 2003), a la cercanía de la Cordillera de Los Andes (Urquiza \& Mella 2002) y a la presencia de árboles nativos (Díaz \& Armesto 2003, Mella \& Loutit 2007). Estos antecedentes permiten afirmar que la predominancia de grandes tamaños prediales con alta cobertura arbórea en jardines de la zona oriente del AMS puede contribuir positivamente a la presencia de avifauna nativa en la ciudad.

E1 conocimiento ecológico debe ser integrado en los procesos de planificación urbana, ya que permite conocer y entender los patrones y procesos que afectan la vegetación y biodiversidad urbana y, a la vez, facilita el diseño de estrategias de conservación en espacios urbanos y periurbanos. Por último, los estudios en grandes ciudades latinoamericanas constituyen un aporte relevante para el conocimiento de patrones y procesos que hasta ahora han sido estudiados principalmente en ciudades del hemisferio Norte.
AGRADECIMIENTOS: Se agradece a la Dirección de Servicios Externos de la Facultad de Arquitectura, Diseño y Estudios Urbanos, Pontificia Universidad Católica de Chile por haber proporcionado la Ortofoto SAF 2006 utilizada para este estudio. Se agradece a los anónimos revisores de la versión preliminar de este artículo ya que sus acertados comentarios contribuyeron a perfeccionarlo.

\section{LITERATURA CITADA}

AKINNIFESI FK, GW SILESHI, OC AJAYI, AI AKINNIFESI, EG DE MOURA, JF LINHARES \& I RODRIGUES (2010) Biodiversity of the urban homegardens of Sao Luís city, Northeastern Brazil. Urban Ecosystems 13: 129-146.

ALBUQUERQUE U, L ANDRADE \& J CABALLERO (2005) Structure and floristics of homegardens in Northeastern Brazil. Journal of Arid Environments 62: 491-506.

ARROYO MTK, R ROZZI, JA SIMONETTI, P MARQUET \& M SALABERRY (1999) Central Chile. In: Mitermeier RA, P Robles Gil \& C Goettsch Mittermeier (eds) Hotspots: Earth's biologically richest and most endangered terrestrial ecosystems: 160-171. Cemex, México DF.

BARBOSA O, J TRATALOS, P ARMSWORTH, R DAVIES, R FUELLER, J PAT \& K GASTON (2007) Who benefits with access from green space? A case study from Sheffield UK. Landscape and Urban Planning 83: 187-195.

BLANCKAERT I, RL SWENNEN, M PAREDESFLORES, R ROSAS-LÓPEZ \& R LIRA-SAADE (2004). Floristic composition, plant uses and management practices in homegardens of San Rafael Coxcatlan, Valley of Tehuacan-Cuicatlan, México. Journal of Arid Environments 57: 39-62.

DANIELS G \& J KIRKPATRICK (2006) Does variation in garden characteristics influence the conservation of birds in suburbia? Biological Conservation 133: 326-335.

DE LA MAZA C, J HERNÁNDEZ, H BOWN, M RODRÍGUEZ \& F ESCOBEDO (2002) Vegetation diversity in the Santiago de Chile urban ecosystem. Arboricultural Journal 26: 347-357.

DÍAZ IA \& JJ ARMESTO (2003) La conservación de las aves silvestres en ambientes urbanos de Santiago. Revista Ambiente y Desarrollo CIPMA (Chile) XIX: 31-38.

ESCOBEDO F, D NOWAK, J WAGNER, CL DE LA MAZA, M RODRÍGUEZ, D CRANE \& J HERNÁNDEZ (2006) The socioeconomics and management of Santiago de Chile's public urban forests. Urban Forestry \& Urban Greening 4: 105-114.

FIGUEROA I (2009) Conectividad y accesibilidad de espacios abiertos urbanos en Santiago, Chile. Tesis de Magíster, Instituto de Estudios Urbanos y Territoriales, Pontificia Universidad Católica de Chile, Santiago.

FORMAN RTT (2008) Urban regions. Ecology and planning beyond the city. Cambridge University Press, Cambridge.

GASTON K, PH WARREN, K THOMPSON \& RM SMITH (2005) Urban domestic gardens IV: The extent of the resource and its associated features. Biodiversity and Conservation 14: 3327-3349.

HERNÁNDEZ J (2007) La situación del arbolado urbano en Santiago. Ambiente Forestal (Chile) 3: 14-16. 
ILUSTRE MUNICIPALIDAD DE SANTIAGO (2006) Plan Estratégico de Desarrollo Comunal. Capítulo I: Diagnóstico Estratégico. Ilustre Municipalidad de Santiago, Secretaría Comunal de Planificación, Dirección de Administración y Finanzas, Santiago.

INE (2004) Censo 2002. Síntesis de resultados. Instituto Nacional de Estadísticas. URL: http://www.ine.cl/ cd2002/sintesiscensal.pdf (accedido 27 diciembre 2011).

JAMES P \& D BOUND (2009) Urban morphology types and open space distribution in urban core areas. Urban Ecosystems 12: 417-424.

KINZIG A, P WARREN, C MARTIN, D HOPE \& M KATTI (2005) The effect of human socioeconomic status and cultural characteristics on urban patterns of biodiversity. Ecology and Society 10: 23 pp. (en línea) URL: www.ecologyandsociety. org/vol10/iss1/art23/ (Accedido 27 diciembre 2011).

KUMAR B \& P NAIR (2004) The enigma of tropical gardens. Agroforestry Systems 61: 135-152.

LORAM A, K THOMPSON, P WARREN \& K GASTON (2008) Urban domestic gardens (XII): The richness and composition of the flora in five cities. Journal of Vegetation Science 19: 321-330.

MARCO A, C BARTHELEMY, T DUTOIT \& V BERTAUDIERE-MONTES (2010) Bridging human and natural sciences for a better understanding of urban floral patterns: The role of planting practices in Mediterranean gardens. Ecology and Society 15: 2 pp. (en línea) URL: http://www.ecologyandsociety.org/vol15/iss2/ art2/ (accedido 27 diciembre 2011).
MELLA J \& A LOUTIT (2007) Ecología comunitaria y reproductiva de aves en cerros islas y parques de Santiago. Boletín Chileno de Ornitología (Chile) 13: $13-27$

MEZA L (2009) Potencialidad de los jardines domésticos urbanos para la conservación de la biodiversidad nativa en Santiago de Chile. Tesis de Magíster, Instituto de Estudios Urbanos y Territoriales, Pontificia Universidad Católica de Chile, Santiago.

MINVU (1992) D.S. N 47 de 1992 Ordenanza General de Urbanismo y Construcciones (actualizada al 13 de Abril del 2011 por el D.S. $N^{\circ} 9$ (V. y U.) del 2011) Ministerio de Vivienda y Urbanismo. URL: http:// www.minvu.cl/opensite_20070404173759.aspx (accedido Agosto 16, 2011).

REYES PAECKE S \& I FIGUEROA (2010) Distribución, superficie y accesibilidad de las áreas verdes urbanas en Santiago de Chile. EURE 109: 89-110.

RODRÍGUEZ J (2008) Movilidad cotidiana, desigualdad social y segregación residencial en cuatro metrópolis de América Latina. EURE 103: 49-71.

SMITH R, K GASTON, PH WARREN \& K THOMPSON (2005) Urban domestic gardens (V): Relationships between landcover composition, housing and landscape. Landscape Ecology 20: 235-253.

URQUIZA A \& J MELLA (2002) Riqueza y diversidad de aves en parques de Santiago durante el periodo estival. Boletín Chileno de Ornitología (Chile) 9: 12-21.

WEZEL A \& S BENDER (2003) Plant species diversity of homegardens of Cuba and its significance for household food supply. Agroforestry Systems 57: 39-49. 